\section{LA CONTRIBUCIÓN DE \\ LAS TIC A LA MEJORA \\ DE LA TRANSPARENCIA \\ ADMINISTRATIVA}

\author{
Agustí Cerrillo-i-Martínez \\ Profesor agregado de Derecho administrativo \\ Estudios de Derecho y Ciencia Política \\ Universitat Oberta de Catalunya \\ Avda. Tibidabo, 39-45. 08015 Barcelona \\ acerrillo@uoc.edu
}

\begin{abstract}
ICTs are bringing about significant changes in the mechanisms by which government transparency is channeled. ICTs enable citizens to participate actively in disseminating information generated using open format data in machine processable form, to interact with one another and with government, and customize public-sector information. ICTs also bolster transparency goals by representing a mechanism that allows greater participation by citizens in government and improved economic and social development. However, to realize these effects and improve government transparency the impact of ICTs on the various mechanisms needs to be regulated and a cultural change fostered in government. Thus, the concept of transparency may itself be affected by ICTs.
\end{abstract}

KEY WORDS: Transparency; ICT; public sector information.

\section{LA TRANSPARENCIA ADMINISTRATIVA EN LA SOCIEDAD DE LA INFORMACIÓN*}

La información del sector público (en adelante, ISP) es la mayor fuente de información en Europa (Comisión Europea, 2009); (OCDE, 2006, 7). Constituye el principal instrumento para conseguir una mayor transparencia administrativa, además de tener un papel significativo en la producción de bienes y servicios de valor añadido.

La transparencia consiste en el conocimiento por parte de los ciudadanos de lo que sucede en el seno de las administraciones públicas. Gracias a la transparencia administrativa las administraciones públicas son vistas

\section{ICTS' CONTRIBUTION TO ENHANCING GOVERNMENT TRANSPARENCY}

RESUMEN: Las TIC están implicando un cambio significativo en los mecanismos a través de los que se canaliza la transparencia administrativa. Las TIC permiten a los ciudadanos participar activamente difundiendo información generada a partir de datos en formatos abiertos y procesables automáticamente, interactuar entre ellos y con las administraciones públicas así como personalizar la información del sector público. Además, las TIC incrementan las finalidades de la transparencia al convertirse en un mecanismo que permite una mayor participación de los ciudadanos en las administraciones públicas y un mayor desarrollo económico y social. Sin embargo, para que estos efectos sean reales y para mejorar efectivamente la transparencia de las administraciones públicas es necesario regular el impacto de las TIC en los diferentes mecanismos e impulsar un cambio de la cultura administrativa. Así pues, el propio concepto de transparencia puede verse afectado por las TIC.

PALABRAS CLAVE: Transparencia; TIC; información del sector público.

como una casa de vidrio (Rivero 1989); (Arena 1996, 15); (Orofino 2005, 3). La quintaesencia de la transparencia es facilitar información a los ciudadanos (Parycek y Sachs 2010, 3).

A lo largo de los últimos años la literatura ha aportado tantas definiciones de transparencia como intentos han habido para definirla (Meijer 2009); (Birkinshaw 2006, 189-191); (Florini 2007, 5); (Piotrowski y Van Ryzin 2007, 306); (Mock 1999, 1082); (Fung 2009); (Chevalier 2007); (Fenster 2010), siendo ésta una noción ni clara ni precisa sino flexible, polisémica y adaptable a múltiples contextos (Blasco Díaz 2010, 126). De la lectura de las diversas definiciones se desprende que la transparencia administrativa se caracteriza por la existencia de información del sector 
público que es puesta a disposición de los ciudadanos a través de diferentes mecanismos para conseguir diversas finalidades. Dicho con otras palabras, la transparencia se puede caracterizar por la existencia de un observador (el ciudadano), un objeto observado (la información del sector público), un medio a través del que se observa (Oliver 2004). Además, la transparencia no es neutra y persigue una finalidad.

En relación a la finalidad de la transparencia, existe un significativo consenso en la literatura sobre el papel de la transparencia administrativa como un instrumento que ha de permitir fortalecer el Estado de Derecho (Sommerman 2010, 20); (García Macho 2010, 30) e incrementar la legitimidad democrática de las administraciones públicas y la rendición de cuentas a los ciudadanos, reduciendo los abusos y la corrupción (Meijer 2009); (Arena 2008, 33); (Moser 2001); (Stiglitz 2007, VII), en la medida en que unos ciudadanos informados pueden participar activamente en las decisiones públicas y controlar la actividad de las administraciones públicas (Sánchez Morón 1991); (Garcia Macho 2010, 34).

También se ha considerado la transparencia como un principio de buena gobernanza (Grimmelikhuijsen 2009, 173); (Curtin 2006); (Frost 2003, 89); (Mock 1999); (Ackerman 2006); (Comisión Europea 2001); (Arena 2008) que puede mejorar el proceso decisional de las administraciones públicas al informar a los ciudadanos sobre los asuntos públicos de manera que su participación pueda ser fundada (Coglianese 2009, 535-536); (Dror 2000); (Gentot 1994); (Sainz Moreno 2004, 165).

Finalmente, la transparencia se ha vinculado a los derechos humanos (Dror 2000, 69); (Birkinshaw 2006).

Pero a pesar de los beneficiosos efectos que se pueden derivar de la transparencia al orientarse a las distintas finalidades que se han identificado, también ha habido quien ha apuntado algunos riesgos o inconvenientes de la extensión de la transparencia al considerar que puede afectar negativamente al proceso decisional y al funcionamiento de las propias administraciones públicas, tener un coste excesivo, así como perjudicar a intereses privados como la propiedad intelectual e industrial, los datos personales o la intimidad (Prat 2006, 101); (Heald 2006a, 60-64); (Lessig 2010, 170).
La existencia de beneficios y ventajas derivadas de la transparencia administrativa, junto con la advertencia de algunos aspectos negativos, lleva a señalar la necesidad de observar que "la relación entre transparencia y secreto no puede resolverse mediante la simple fórmula del principio de transparencia absoluta" (Sainz Moreno 2004, 165), por lo que será necesario apuntar ciertos límites a la transparencia fundados en la necesidad de proteger otros intereses tanto públicos (determinadas políticas públicas -exterior, defensa, seguridad pública, monetaria-, eficacia administrativa en la toma de decisiones) como privados (datos personales, privacidad, secreto comercial, etcétera).

En los últimos años, la extensión de las tecnologias de la información y la comunicación (en adelante, TIC) ha facilitado la evolución de las funciones y los mecanismos a través de los que se canaliza la información del sector público y, por ende, la transparencia (Fung, Graham y Weil 2007, 25); (Cerrillo i Martínez 2005); (Cavaleri y Venturini 2004, 11); (Ackerman 2006, 125); (Fenster 2010, 668). De hecho, está ampliamente recogido en la literatura que actualmente la transparencia se caracteriza generalmente por canalizarse a través de medios electrónicos (Cerrillo i Martínez 2005); (Curtin 2006); (Grimmelikhuijsen 2009, 175); (Meijer 2009, 258).

Las TIC están teniendo un impacto significativo en las diferentes características que definen la transparencia administrativa. Este impacto está siendo asumido de manera progresiva por las administraciones públicas que han ido impulsando la aprobación de diversas normas e implementado diferentes políticas públicas para reflejar algunos de los cambios que se pueden derivar del uso intensivo de las TIC para canalizar la transparencia administrativa. Además la propia ciudadanía también está contribuyendo a este proceso accediendo cada vez con mayor intensidad a los espacios a través de los que se difunde información del sector público y participando activamente en la difusión de información del sector público contribuyendo de este modo a incrementar la transparencia

Precisamente, el presente artículo se propone analizar el impacto que las TIC tienen en la transparencia administrativa y valorar como éstas inciden en las diferentes características de la transparencia. 
A tal fin se analizará la evolución de la regulación de los diferentes mecanismos a través de los que se canaliza la transparencia administrativa en España y de diferentes políticas públicas vinculadas a la transparencia administrativa. Asimismo, en la medida en que los pocos estudios y análisis que se han hecho en nuestro pais lo permitan, se expondrá su aplicación efectiva. Además, se incluyen referencias comparadas sobre diferentes normas y políticas públicas que están siendo implementadas en los últimos años por diversos países occidentales para mejorar la transparencia administrativa a través del uso de las TIC.

\section{LOS MECANISMOS DE TRANSPARENCIA ADMINISTRATIVA Y SU EVOLUCIÓN}

La evolución de la transparencia administrativa en España está estrechamente vinculada al desarrollo de nuestro sistema democrático (Mestre Delgado 1998, 46).

Desde la aprobación de la Constitución en 1978, que implícitamente incorpora el principio de transparencia a través del reconocimiento del derecho de acceso a archivos y registros administrativos (artículo 105.b), diferentes normas han regulado diversos mecanismos a través de los cuales los ciudadanos pueden tener conocimiento de la información del sector público y numerosas administraciones públicas han puesto en marcha los mecanismos necesarios para garantizar el pleno ejercicio y uso de dichos mecanismos.

El artículo 3 Ley 30/1992, de 26 de noviembre, de régimen jurídico de las administraciones públicas y del procedimiento administrativo común (en adelante, LRJPAC), desde su reforma de 1999, así como diferentes leyes autonómicas en esta materia reconocen la transparencia como principio de la actuación de las Administraciones públicas (por ejemplo, el artículo 31 Ley 26/2010, de 3 agosto, de Régimen jurídico y de procedimiento de las administraciones públicas de Cataluña). También las pocas leyes que se han aprobado en España sobre administración electrónica siguiendo lo previsto con carácter básico en el artículo 3 Ley $11 / 2007$, de 22 de junio, de acceso electrónico de los ciudadanos a los servicios públicos (en adelante, LAECSP), incorporan este principio'.
En la actualidad existen numerosos mecanismos a través de los que se canaliza la transparencia administrativa ${ }^{2}$. Los diferentes mecanismos existentes tienen funciones diferentes, aunque un papel complementario (Merloni 2008,

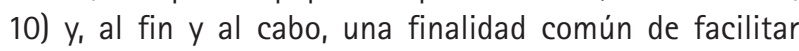
el conocimiento público de la información del sector público.

El alcance de los diferentes mecanismos a través de los que se canaliza la transparencia ha evolucionado en los últimos años por el uso intensivo de las TIC que están constituyendo un motor útil en aras a incrementar la transparencia de nuestras administraciones públicas. Incluso la extensión de las TIC está facilitando el surgimiento de nuevos mecanismos. Precisamente, en las próximas páginas se centrará la atención en el impacto que las TIC están teniendo en los diferentes mecanismos a través de los que se canaliza la transparencia administrativa.

\subsection{El derecho de acceso a los archivos y registros administrativos}

El derecho de acceso a los archivos y registros administrativos es el mecanismo a través del cual los ciudadanos pueden tener conocimiento de la información administrativa previa solicitud a la administración pública para que se la muestre o les facilite una copia.

El artículo 105.b) Constitución ya previó en 1978 que la ley regularía el acceso de los ciudadanos a los archivos y registros administrativos. Sin embargo, éste no fue regulado hasta la aprobación de la LRJPAC en 1992, cuyo artículo 35 recoge entre los derechos de los ciudadanos el de acceso a los archivos y registros administrativos que fue desarrollado por el artículo 37 LRJPAC. Desde la aprobación de la LRJPAC la regulación del derecho de acceso ha sido objeto de un amplio análisis (Cerrillo i Martínez 2000); (Fernández Ramos 1997); (Mestre Delgado 1998); (Rams Ramos 2008) pero también de una fuerte crítica (Parada Vázquez 1993, 108).

Posteriormente, esta norma ha sido objeto de desarrollo por parte de diferentes Comunidades Autónomas que sin introducir novedades relevantes han incluido referencias a este derecho en su normativa sobre administraciones públicas o sobre archivos administrativos. Asimismo, en ámbitos sectoriales como el medio ambiente, este derecho

ARBOR Vol. 188756 julio-agosto [2012] 707-724 ISSN: 0210-1963

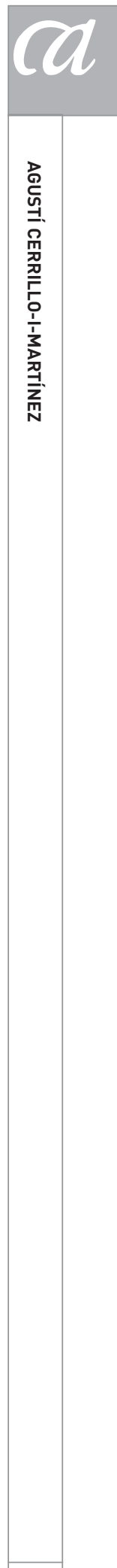

709 
ha sido objeto de una regulación específica que, en algunos aspectos, ha supuesto una mejora sensible de la regulación básica. Así, por ejemplo, con la finalidad de trasponer la Directiva 98/2003, se aprobó la Ley 27/2006, de 18 de julio, por la que se regulan los derechos de acceso a la información, de participación pública y de acceso a la justicia en materia de medio ambiente (Pigrau Solé 2008); (Razquin Lizarraga y Ruiz de Apodaca Espinosa 2007).

A grandes rasgos, las diferentes regulaciones se basan en el reconocimiento de un derecho subjetivo, que goza de tutela judicial, por el que el ciudadano sin necesidad de mostrar ningún interés solicita a la administración pública visualizar un documento administrativo. En principio, la LRJPAC prevé el acceso respecto a documentos que obren en los archivos administrativos y formen parte de un expediente que corresponda a un procedimiento terminado, lo que ha sido ampliamente criticado. Sin embargo, posteriormente, la Ley 27/2006 delimita el ámbito objetivo en relación a la información sobre medio ambiente.

El acceso a los documentos persigue facilitar que los ciudadanos puedan tener conocimiento de la actividad administrativa a posteriori. De hecho, el acceso a los documentos administrativos se conoce como un mecanismo para la transparencia ex post o reactivo que exige a las administraciones públicas únicamente facilitar información a los ciudadanos cuando éstos se la solicitan, lo que lo convierte más en un mecanismo para controlar la actividad administrativa y exigir la rendición de cuentas (Mock 1999, 1097).

Precisamente, que el derecho de acceso esté basado en una petición (request-and-wait-for-a-response-approach) constituye su talón de Aquiles (Herz 2009, 585); (Vladeck 2008, 1789). Como pone de relieve Herz, este enfoque es muy limitado en la medida en que el solicitante no tiene conocimiento de lo que tiene la administración pública $y_{\text {, }}$ por lo tanto, qué es lo que puede pedir.

El uso de las TIC puede tener un impacto positivo en el derecho de acceso tanto facilitando la solicitud de acceso y la visualización de los documentos por parte del solicitante, como garantizando la buena gestión y archivo de los documentos que faciliten su puesta a disposición de los ciudadanos. Sin embargo, actualmente únicamente la Ley 27/2006 reconoce el papel que las TIC pueden tener para canalizar el acceso a la información sobre medio ambiente (artículos 5 y 6).

A pesar de las diferentes regulaciones, el uso del derecho de acceso a los documentos administrativos en España es significativamente bajo como se deriva de los pocos estudios realizados ${ }^{3}$. Un motivo lo constituye la regulación restrictiva del derecho de acceso, hasta el extremo que en algunos estudios internacionales no se incluye a España entre los paises que tienen en vigor una norma sobre acceso a la información (Mendel 2008). Otro, el impacto de las TIC en este derecho que, por un lado, lo ha hecho obsoleto por estar basado en la petición individualizada de información en soporte papel (Vladeck 2008, 1792-1793) y, por otro, no se ha facilitado su uso al no haberse incorporado el mismo en la regulación del derecho de acceso.

Desafortunadamente, esta situación es bastante generalizada en todo el mundo donde se han aprobado más de 85 leyes sobre acceso a la información (Michener 2009); (OCDE 2001); (Ackerman 2006, 26); (Mendel 2008) aunque su aplicación haya sido más bien limitada (Frost 2003); (Privacy International 2006); (Molnar 2009); (Hazell y Worthy 2010); (Cluzel-Métayer 2006, 253-254); (Carloni 2005); (Apfelroth 2006).

Sin embargo, en algunos paises se han ido introduciendo algunas modificaciones tanto en la regulación del acceso a la documentación como en su implementación para incrementar su uso por parte de los ciudadanos, para impulsar la modernización administrativa que acentúa la transparencia y la apertura y recoger el impacto de las TIC que han de permitir a las administraciones públicas encontrar, compartir, combinar y reutilizar los contenidos públicos (Dawes 2010, 378). Entre otros se podría traer a colación la regulación de las instituciones de la Unión Europea (Reglamento 1049/2001 del Parlamento Europeo y del Consejo, de 30 de mayo de 2001, relativo al acceso del público a los documentos del Parlamento Europeo, del Consejo y de la Comisión), el Convenio del Consejo de Europa sobre acceso a los documentos oficiales (2008), el Código de administración electrónica italiano (2005), la Ley francesa que incorpora diferentes medidas para mejorar las relaciones entre la administración y los ciudadanos (2005) o la modificación de la Freedom of Information Act norteamericana (1996). 
Asimismo, algunos países también han impulsado políticas públicas para mejorar el acceso a la información del sector público. Tal vez la que ha tenido una mayor relevancia se contiene en el Memorando emitido por el presidente Barack Obama el 21 de enero de 2009 en el que se indica que "la Ley de acceso a la información debe ser aplicada con una clara presunción: en caso de duda, prevalece la apertura" ${ }^{4}$. De todos modos, como han apuntado algunas instituciones a pesar de los cambios no ha habido un incremento significativo de la transparencia pública sino más bien al contrario (Openthegovernment.Org 2010, 1-3).

En España, siguiendo la estela de estas reformas, en los últimos años ha habido algunos movimientos a favor de una modificación sustancial de la regulación de este derecho hasta el punto de que en 2010 el gobierno impulsó un proceso para la elaboración de un proyecto de ley que, finalmente, no prosperó por la disolución de las Cortes en septiembre de $2011^{5}$. Posteriormente, en abril de 2012, se ha sometido al trámite de información pública el anteproyecto de ley de transparencia, acceso a la información y buen gobierno. El anteproyecto realiza una definición más amplia del objeto del derecho (la información pública) y perfila mejor los límites y excepciones al mismo. Asimismo, se prevé que el acceso a la información se realizará preferentemente por vía electrónica ${ }^{6}$. Finalmente, se prevé la reclamación ante la Agencia Estatal de Transparencia, Evaluación de las Políticas Públicas y de la Calidad de los servicios frente a toda resolución en materia de acceso.

De todos modos, como afirma Roberts "en la práctica, la probabilidad que aprobando una ley sobre el acceso a la información conducirá a un cambio cultural o incrementará la confianza es pequeña" (Roberts 2006, 118); (OECD 2010, 4). Por ello, será importante articular alrededor de esta norma otros instrumentos que faciliten la consecución de sus finalidades.

\subsection{La difusión de información del sector público}

Un segundo mecanismo de transparencia administrativa que ha ido adquiriendo una importancia significativa en los últimos años gracias al uso de las TIC es la difusión de información (Darbishire 2010, 9).

La difusión de la información del sector público consiste en la divulgación generalizada de información mediante la creación de servicios de difusión de la información por parte de los poderes públicos (Cerrillo i Martínez 2005, 14); (Cerrillo i Martínez 2011). A diferencia del derecho de acceso, la difusión de información no sólo constituye un mecanismo de control de la actividad de las administraciones públicas sino que también permite que los ciudadanos y las empresas conozcan la información pública necesaria tanto para su quehacer diario como para el ejercicio de sus derechos así como el cumplimento de sus obligaciones, realizar sus actividades y disfrutar de los servicios públicos, conocer la actividad que realizan las administraciones públicas $y_{1}$ en general, participar en la vida pública. De este modo, la difusión de información por parte de las administraciones públicas permite que los ciudadanos conozcan en tiempo real la acción administrativa y puedan aprovecharse de la información del sector público. Nos encontramos, así, ante un mecanismo ex ante y proactivo de transparencia lo que significa que la actividad de las administraciones públicas puede ser continuamente difundida por ellas y conocida por los ciudadanos (Cerrillo i Martínez 2005, 14); (Heald 2006b, 32); (Mattarella 2005, 12 y ss.); (Merloni 2005, 127 y ss.); (Kubicek 2004, 279 y ss.); (Sucevic 2008, 114).

Los portales de las administraciones públicas en Internet, su presencia en las redes sociales como Facebook, Second Life o Twitter o el uso de blogs y wikis constituyen algunos ejemplos del uso de las TIC por las administraciones públicas para difundir información del sector público.

La regulación del uso de las TIC en la difusión de información pública en España no se da hasta la aprobación de la Ley 30/1992, de 26 de noviembre, de régimen jurídico de las Administraciones públicas y del procedimiento administrativo común. Sin embargo, no existe actualmente en España una regulación de la difusión de información del sector público sino un conjunto disperso de preceptos $^{7}$. Algunos se limitan únicamente a establecer normas relativas a aspectos organizativos vinculados a la difusión de información pública. Otros llegan a establecer principios generales y reconocer derechos de los ciudadanos a obtener información pública también a través de medios electrónicos (Cerrillo i Martínez 2010b).

La aprobación de la Ley 11/2007, de 22 de junio, de acceso electrónico de los ciudadanos a los servicios públicos (LAECSP) ha supuesto un importante paso adelante en la regulación del uso de las TIC por parte de las administra-

ARBOR Vol. 188756 julio-agosto [2012] 707-724 ISSN: 0210-1963 
ciones públicas, aunque no regula de manera especifica la difusión de información pública a través de medios electrónicos al regular los principios de la administración electrónica, los derechos de los ciudadanos y la sede electrónica (Valero Torrijos 2009). De todos modos, aún es necesario regular con mayor detalle este mecanismo. Son expresivas al respecto las palabras de Cotino, "[p]ero en casa del herrero, cuchara de palo: los poderes públicos no se obligan jurídicamente a ellos mismos a facilitar información pública por medios electrónicos ni facilitar la participación y apertura (Cotino Hueso 2007, 92).

El anteproyecto de ley de transparencia, acceso a la información y buen gobierno incorpora una regulación mucho más exhaustiva de la difusión de información del sector público que la legislación vigente, definiendo la obligación de las administraciones públicas de difundir de forma periódica y actualizada aquella información cuyo conocimiento sea relevante para garantizar la transparencia de su actividad. También define la información que necesariamente deberá ser publicada por las administraciones públicas (información institucional, organizativa y de planificación, información de relevancia jurídica, información económico-presupuestaria e información estadística). Finalmente, prevé la creación de un Portal de Transparencia por parte de la Administración General del Estado.

Asimismo, diferentes normas autonómicas y locales han desarrollado los preceptos básicos contenidos en la LAECSP (Cerrillo i Martínez 2010a y Cerrillo i Martínez 2009a). En particular, resulta de interés traer a colación la Ley 29/2010, de 6 de agosto, de uso de los medios electrónicos en el sector público de Cataluña, en la que se reconoce el carácter de servicio público de la difusión de la información general del sector público que se presta mediante la correspondiente sede electrónica (artículo 11.1) y se define qué se entiende por información del sector público de calidad (artículo 9) 7 bis.

Así, en términos generales, se puede considerar la difusión de información del sector público como un servicio público. Como afirma Malaret, "es precisamente en este contexto de aportar garantías y seguridades ante los nuevos retos que tenemos planteados ciudadanos y administraciones públicas, que parece pertinente utilizar un concepto jurídico que nos permita referirnos a todo un haz de derechos de los ciudadanos y obligaciones de las administraciones públicas" (Malaret I García 2007, 174); (Alonso Espinosa 2008, 46); (Martínez Gutiérrez 2009, 466). Pero de todos modos sería deseable un mayor reconocimiento legislativo de este servicio $y$, por ende, de la obligación de las administraciones públicas de difundir información tal y como ha hecho, por ejemplo, el legislador catalán, para proporcionar una mayor seguridad jurídica y el poder de determinar las condiciones bajo las que debe ser proporcionada la información a los ciudadanos a través de las TIC.

A diferencia del derecho de acceso, las TIC son ampliamente utilizadas por los ciudadanos para conocer la información del sector público. Por un lado, se pueden traer a colación los pocos datos disponibles públicamente sobre accesos a los portales de las administraciones públicas ${ }^{8}$.

Por otro lado, resultan de interés los resultados del Barómetro de septiembre de 2009 del CIS, en el que se observa que entre los usuarios españoles de Internet en los últimos doce meses (55,8\% de la población), un $35,6 \%$ lo habian utilizado, entre otros, para realizar gestiones con las administraciones públicas con una frecuencia alta 0 muy alta. Previamente, el Barómetro de marzo de 2009 había analizado el uso que los españoles hacian de la administración electrónica observando que el $71,8 \%$ de los encuestados conocian la posibilidad de utilizar estas tecnologías en sus relaciones con las administraciones públicas $y$, en particular, un 8,9\% de ellos conocía la posibilidad de obtener información sobre distintas cuestiones generales. Entre las diferentes tecnologías de la información y la comunicación, los ciudadanos prefieren Internet $(19,7 \%)$ frente al teléfono $(6,7 \%)$ o el fax $(0,3 \%)$. En general, entre los usos de los ciudadanos que han realizado algún trámite con la administración pública a través de Internet, el principal $(50,7 \%)$ es para obtener información sobre los requisitos o procedimiento para realizar una gestión, encontrando el 89,3\% lo que buscaba, un $77,9 \%$ de manera fácil o muy fácil y el $80,7 \%$ quedando muy satisfechos.

Comparativamente, se puede observar cómo algunas de las normas que regulan el acceso a la información han incluido referencias a la difusión de información del sector público $y$, en particular, la obligación de difundirla (Access Info Europe y Open Knowledge Foundation 2010, 20); (Darbishire 2010). 
Así puede traerse a colación de nuevo la Freedom of Information Act estadounidense que, desde su modificación de 1996, prevé que se difundirá a través de las salas electrónicas de lectura aquellos documentos que tengan suficiente interés general y que hayan sido solicitados por más de tres personas (Herz 2009, 587) o la Ley sobre administración electrónica (e-Government Act) que define las características de la información que se difunde a través de los portales de la administración federal. Asimismo, la legislación francesa ha previsto, en su modificación de 2005, que el derecho de acceso no se aplica cuando previamente se ha difundido un documento administrativo de modo que se agiliza y disminuye la carga de trabajo de las administraciones públicas (Puybasset 2003, 1307). Igualmente el Código de administración electrónica italiano (Carloni 2008).

Además también se han ido impulsando diferentes políticas públicas con el objetivo de avanzar en el uso de las TIC para difundir la información del sector público $y$, por ende, incrementar la transparencia administrativa. Tal vez, el caso que ha tenido una mayor trascendencia y repercusión se ha dado nuevamente en los Estados Unidos de América a partir del Memorando sobre Transparencia y Gobierno Abierto impulsado por Barack Obama en el que se establece que "los departamentos ejecutivos y las agencias deben utilizar las nuevas tecnologías para poner información sobre sus actividades y decisiones en línea y fácilmente disponible para los ciudadanos". En desarrollo de estos planteamientos, se aprobó la Directiva sobre el Gobierno Abierto en diciembre de 2009 que prevé que "cada agencia debe adoptar pasos rápidos para ampliar el acceso a la información haciéndola disponible en línea en formatos abiertos".

\subsection{La reutilización de la información del sector público}

En los últimos años la extensión de las TIC está facilitando la generación de informaciones de valor añadido y la oferta de servicios basados en información del sector público.

La reutilización de la información del sector público permite que las empresas y los ciudadanos puedan añadir valor a la información del sector público ofreciendo al mercado y, en general, a la sociedad, nueva información o servicios basados en la misma. A pesar de que en un primer momento se había vinculado este mecanismo con la comercialización de la información del sector público, con el tiempo se ha ido destacando también el potencial que tiene como mecanismo para canalizar la transparencia administrativa. A través de la reutilización de la información del sector público se puede incrementar la transparencia administrativa en la medida en que se da una mayor difusión a información del sector público o información basada en la misma.

La información del sector público tiene un papel significativo como motor no sólo económico sino también transnacional y, por ello, la Comisión Europea ha ejercido un rol relevante en el fomento de su reutilización, así como fijando unas reglas del juego que eliminen los obstáculos que existen en el mercado interior de la reutilización como las prácticas discriminatorias, los mercados monopolísticos basados en acuerdos exclusivos y la falta de transparencia (Comisión Europea 1998); (Comisión Europea 2009).

En este ámbito, sobre la base de la Directiva 2003/98/ CE se ha aprobado la Ley 37/2007, de 16 de noviembre, de reutilización de la información del sector público (en adelante, LRISP) (Cerrillo i Martínez 2009b).

La reutilización consiste en el uso de documentos que obran en poder de las entidades del sector público, por personas físicas o jurídicas, con fines comerciales o no comerciales, siempre que dicho uso no constituya una actividad administrativa pública (artículo 3 LRISP). La definición de la reutilización que hace la LRISP es clara y permite diferenciarla netamente de otros usos de la información del sector público a los que se ha hecho referencia con anterioridad ${ }^{9}$. Sin embargo, introduce la duda de si, a través de la reutilización, terceras personas pueden difundir información a través de medios electrónicos a fin de incrementar la transparencia administrativa en la medida en que ésta constituye una actividad administrativa pública. En este punto, el recurso a la directiva que se refiere a los usos "distintos del propósito inicial que tenían esos documentos en la misión de servicio público para la que se produjeron" y no lo circunscribe a los límites fijados por el legislador español lo que lleva a concluir que no debe existir obstáculo alguno para tal fin.

ARBOR Vol. 188756 julio-agosto [2012] 707-724 ISSN: 0210-1963 
Sin embargo, la LRISP no establece una obligación de reutilizar la información del sector público dejando en manos de cada administración la decisión sobre esta cuestión (artículo 4).

La LRISP prevé diferentes instrumentos mediante los que autorizar y fijar las condiciones a través de las que se realice la reutilización. También apunta la posibilidad de extender el uso de las TIC tanto para facilitar la tramitación de las licencias-tipo a través de las cuales se autorice la reutilización, así como para facilitar la reutilización (creación de listados e índices accesibles en línea de los documentos disponibles, puesta a disposición de documentos por medios electrónicos en los formatos o lenguas preexistentes) (artículos 4 y 5 LRISP).

No existen cifras sobre el volumen de información del sector público que se reutiliza en España aunque existen diferentes muestras de que esta actividad está creciendo significativamente impulsada tanto por empresas como por ciudadanos. Por un lado, el valor del mercado de la información del sector público de la Unión Europea se estima entre 27.000 (Dekkers, Polman, Velde y Vries 2006) y 68.000 miIlones de euros (Pira International 2000). Por otro lado, los diferentes indicadores utilizados para medir la reutilización de la información del sector público ponen de manifiesto un crecimiento del mercado y un aumento de la reutilización en diferentes sectores durante los últimos años según un estudio que impulsó la Comisión Europea para evaluar el impacto de la Directiva en tres sectores principales de la información del sector público (información geográfica, meteorológica y jurídico-administrativa). Así, por ejemplo, por lo que se refiere a la información geográfica, los volúmenes de descarga de información del sector público crecieron aproximadamente un 350\% entre 2002 y 2007. Por lo que respecta a la información meteorológica, según los institutos nacionales de meteorología, los volúmenes de descarga han aumentado un 70\% entre 2002 y 2007 (Fornefeld, Boele-Keimer, Recher y Fanning 2009).

Sin embargo, aún existen problemas de orden práctico para la reutilización de la ISP. Por ejemplo, la Comisión Europea apunta la falta de información sobre los datos disponibles acerca del sector público, la falta de interés de los organismos del sector público de reutilizar comercialmente su información o la falta de consciencia sobre su potencial económico (Comisión Europea 2009).
En cualquier caso, el mercado europeo de la información del sector público aún está lejos del norteamericano donde la información del sector público es ampliamente reutilizada. Los Estados Unidos de América tienen una larga experiencia en relación a las políticas activas de difusión y comercialización de la información del sector público (Ramos Simón 2003, 69). La legislación norteamericana se caracteriza por no prever un copyright gubernamental ni restricciones a la reutilización y por establecer unos precios máximos de la información que se limita a los costes marginales de reproducción y difusión. Ello ha permitido que el mercado norteamericano de la información del sector público haya sido durante tiempo hasta cinco veces mayor al comunitario (Pas 2002).

\subsection{La apertura de datos públicos y el gobierno abierto}

La apertura de datos públicos constituye una evolución facilitada por las TIC de la reutilización de la información del sector público que fija la atención no tanto en garantizar las condiciones necesarias para que la información sea reutilizada como en que las administraciones públicas faciliten información que pueda ser fácilmente reutilizada.

Este mecanismo comparte algunas finalidades y regulaciones con los anteriores aunque intenta superar algunos de los límites, básicamente los derivados de la propia actitud de las administraciones públicas frente a la difusión de la información y da un mayor protagonismo a los ciudadanos. En cualquier caso, está ampliamente reconocido que a través de la apertura de datos públicos se favorece la transparencia administrativa en la medida en que se facilita la reutilización de los mismos para analizar y evaluar la actividad administrativa (Access Info Europe y Open Knowledge Foundation 2010, 29). En esta misma línea, Tim Berners-Lee, uno de los principales impulsores de la apertura de datos en Gran Bretaña, afirma al respecto que "los datos de las administraciones públicas se están poniendo en línea para aumentar la rendición de cuentas, facilitar información útil sobre el mundo, y para que las administraciones públicas del país y el mundo funcionen de manera más eficiente" (Berners-Lee 2009).

Según está ampliamente reconocido, los datos públicos son aquellos datos objetivos, fácticos, no personales sobre 
los que los servicios públicos funcionan y con los que posteriormente son evaluados, en los que se basan las decisiones públicas o los que son recogidos o generados en el curso de la prestación de servicios públicos ${ }^{10}$. Con este planteamiento se superan tanto las nociones de información como de documento que caracterizan el resto de mecanismos de transparencia administrativa.

Los datos públicos que se pongan a disposición de los ciudadanos deben cumplir determinadas características recogidas en los Principios de los datos abiertos que persiguen, precisamente, garantizar la fácil reutilización de los mismos. En particular, los datos deben ser completos, primarios, oportunos, accesibles, procesables automáticamente, no discriminatorios, no propietarios, sin licencia ${ }^{11}$. Asimismo, existen otros aspectos que deben tenerse en cuenta cuando se liberan los datos como son la facilidad para su localización o el coste vinculado a su reutilización (Access Info Europe y Open Knowledge Foundation 2010, 42).

Sin embargo, a pesar de que el proceso de apertura de datos públicos fija su foco en las administraciones públicas, debe destacarse, siguiendo a Llinares, que el espíritu de la filosofía de open data es "coger datos que en sí mismos no ofrecen ningún valor y dotarlos de significado para convertirlos en conocimiento válido y con valor para los demás" (Llinares 2010, 62). Es decir, la conjugación entre administraciones públicas y ciudadanos para dar la mayor difusión posible a la información del sector público. Ello pasa por que los datos públicos sean fáciles de localizar, de licenciar y de reutilizar.

Buena parte de los proyectos de apertura de datos públicos que se están impulsando tienen una perspectiva más amplia vinculándolos al movimiento del gobierno abierto (open government) ${ }^{11}$ bis. Al respecto, de acuerdo con Calderón y Lorenzo "nos estamos refiriendo a una evolución de nuestro sistema democrático de convivencia y valores basada en el establecimiento de mecanismos para la transparencia de los gobiernos así como de espacios permanentes de colaboración y participación de los ciudadanos más allá del ejercicio de derecho de sufragio cada cuatro años" (Calderón y Lorenzo 2010, 11). Desde esta perspectiva, la apertura de datos constituye la base para el gobierno abierto en la medida en que sólo de este modo a través de un acceso fácil y libre a la información del sector público se puede favorecer la participación y la colaboración (Parycek y Sachs 2010, 3).

La vinculación de la apertura de datos con la transparencia resulta clara. Como destaca el informe de seguimiento del Memorando sobre Transparencia y Gobierno Abierto impulsado por Barack Obama, la apertura promueve la rendición de cuentas del gobierno y también el intercambio de información pública haciéndola disponible en formatos abiertos y accesibles. Pero no se puede olvidar que el gobierno abierto también implica la participación y colaboración ciudadanas ${ }^{12}$.

Poco a poco van extendiéndose los proyectos de apertura de datos públicos ${ }^{13}$. Una de las iniciativas pioneras en España se desarrolla de la mano del gobierno vasco, quien ha puesto en marcha un proyecto de apertura de datos públicos con el objetivo, entre otros, de aumentar la transparencia. A través de un portal se ponen a disposición de los ciudadanos datos públicos bajo licencias de propiedad abiertas que permitirán su redistribución, su reutilización y su comercialización ${ }^{14}$.

Otra experiencia es el sitio del Gobierno del Principado de Asturias, donde se encuentran publicados conjuntos de datos de carácter público. Aunque en la actualidad el número de datos difundidos es bajo, este portal constituye un instrumento útil para facilitar tanto el aprovechamiento de los datos de carácter público de la forma más efectiva, como la transparencia de la administración ${ }^{15}$. También se puede traer a colación la experiencia de Castilla y León o de Cataluña o, a nivel local, de los ayuntamientos de Gijón, Zaragoza o Barcelona. Finalmente, en noviembre de 2011, la Administración General del Estado ha puesto en funcionamiento su portal.

En el ámbito internacional existen otras experiencias de apertura de datos públicos. Así, por ejemplo, los Estados Unidos de América (data.gov) y Reino Unido (data.gov.uk) han puesto en sus sitios web cientos de miles de conjuntos de datos en formatos procesables automáticamente.

La apertura de datos públicos no ha sido objeto, hasta el momento, de un reconocimiento normativo más específico que el que se deriva de la regulación de la reutilización de la información del sector público. Siguiendo los principios propios de la normativa sobre reutilización de la informa- 
ción del sector público, cada proyecto define las condiciones específicas a las que se somete la reutilización a través de cláusulas recogidas en los propios portales, mediante el establecimiento de licencias de uso o la previsión de un código de conducta ${ }^{16}$. Estas condiciones pueden regular cuestiones relativas a los usos que se pueden realizar de los datos (comercial o no comercial); la propiedad intelectual de los mismos; las condiciones de la reutilización (reconocimiento de la autoría o cita de la fuente, por ejemplo); la calidad de los datos y la responsabilidad sobre los daños generados por la consulta de los mismos; la protección de datos personales o la seguridad de los datos.

\section{El impacto de las TIC EN LA tRANSPARENCIA ADMINISTRATIVA: HACIA UNA TRANSPARENCIA $\mathbf{2 . 0}$ DE CALIDAD}

Las TIC están incidiendo en los diferentes mecanismos a través de los que se canaliza la transparencia administrativa afectando a las características que la definen (sujetos, objeto, medios y finalidades).

Por lo que se refiere al sujeto, tradicionalmente la transparencia se ha caracterizado por la puesta a disposición de los ciudadanos por las administraciones públicas de información del sector público. En la actualidad, el desarrollo tecnológico está facilitando que los propios ciudadanos contribuyan a incrementar la transparencia administrativa dejando de ser responsabilidad exclusiva de las administraciones públicas (Acar, Alonso y Novak 2009).

En particular, las tecnologías 2.0 están facilitando que los ciudadanos, más allá de ser meros observadores de la información del sector público, difundan información del sector público $y$, además, puedan interaccionar tanto con las administraciones públicas como con otros ciudadanos en este proceso (Mayo y Steinberg 2007). Así, la extensión de las tecnologías 2.0 sitúa a los ciudadanos no sólo como receptores de información del sector público difundida por las propias administraciones sino como generadores y difusores de la misma. La naturaleza viral de estas tecnologías facilita y acelera la difusión de información del sector público (Chang y Kannan 2008, 20), así como la generación de contenido por los propios usuarios (OCDE 2007). Se avanza hacia lo que algunos han Ilamado ciudadanos 2.0
(Bae Brandtzæg 2010). En este sentido, Fung, Graham y Weil apuntan que está emergiendo un nuevo estadio en relación a los mecanismos de transparencia administrativa en la que los ciudadanos son autorizados para facilitar y compartir información (Fung et al. 2007, 169). De hecho, esta posibilidad traslada a la administración pública el principio propio de la ingeniería de separar los datos de su interacción, que se utiliza comúnmente en la construcción de sitios web (Robinson, Yu, Zeller y Felten 2009, 161).

Respecto al objeto, el análisis realizado en las páginas anteriores permite observar que las TIC han propiciado una evolución del objeto de la transparencia que transita desde el reconocimiento del acceso a archivos y registros hasta la puesta a disposición de los ciudadanos de datos en formatos abiertos y procesables automáticamente a fin de que sean reutilizados. Sin embargo, esta evolución no ha ido generalmente acompañada de una concreción del alcance del objeto en relación a los diferentes mecanismos.

Asimismo, debe tenerse en cuenta que no cualquier información, documento o dato permite mejorar la transparencia administrativa. Es necesario que el objeto sobre el que se basa la transparencia sea de calidad (Cerrillo i Martínez y Galán Galán 2007). Éste es un aspecto particularmente importante en aquellos casos en que sean los ciudadanos los que canalicen la transparencia administrativa, puesto que si bien la administración pública es responsable de la veracidad y de la integridad de la información (art. 4 LAECSP), no debe ocurrir exactamente lo mismo cuando la misma información está siendo difundida por los ciudadanos. Por ello, como observa Meijer, la transparencia mediada tecnológicamente puede amenazar a la confianza en las administraciones públicas (Meijer 2009, 266). Cuando la transparencia es canalizada por las propias administraciones públicas, ya se ha apuntado anteriormente la importancia de difundir información del sector público de calidad tal y como así se deriva del ordenamiento jurídico (por ejemplo, artículos 4 y 6 LAECSP). Sin embargo, cuando son los ciudadanos los que participan en la difusión de información del sector público puede ser útil utilizar otros instrumentos para tal fin como el establecimiento de códigos de conducta y condiciones de reutilización que deberá concretar en este sentido cada administración pública al poner a disposición de los ciudadanos informaciones 0 datos para su reutilización, así como arbitrar instrumentos que permitan informar sobre la incorrección de la informa- 
ción o la falta de calidad para evitar errores o confusiones así como la corrección o actualización inmediata de la información que no sea de calidad (Cerrillo i Martínez 2012). Asimismo, para facilitar la transparencia no sólo es necesario que las administraciones públicas difundan información del sector público sino también que lo hagan de manera estructurada, abierta y procesable para que pueda ser fácilmente reutilizada (Guadián 2010, 84).

Por lo que respecta a los medios, tal y como se ha apuntado anteriormente, el desarrollo tecnológico no sólo permite una amplia difusión de la información entre los ciudadanos sino también que una pluralidad de sujetos participen en la difusión de la información, que puedan interactuar entre ellos así como personalizar la información del sector público que se difunde en función de su destinatario. De este modo las TIC están suponiendo un cambio significativo en los mecanismos a través de los que se canaliza la transparencia administrativa no sólo mejorando su eficacia y eficiencia sino también afectando significativamente al propio concepto.

Finalmente, desde la perspectiva de las finalidades de la transparencia administrativa, las TIC las han ampliado sensiblemente al facilitar la evolución desde las finalidades clásicas vinculadas a la mejora de la legitimidad de las administraciones públicas, así como al control sobre la actuación administrativa, para convertirse también en un mecanismo a través del cual se puede conseguir una mayor participación de los ciudadanos en los servicios públicos $y_{1}$ en general, en las administraciones públicas, así como un mayor desarrollo económico y social gracias no sólo a la reutilización de la información del sector público sino también a su difusión.

Sin embargo, a pesar del impacto evidente de las TIC en la transparencia administrativa, éstas no suponen en todo momento una mejora de la misma. Existen diferentes riesgos vinculados a la extensión de las TIC que pueden incidir negativamente en la transparencia administrativa. Tal vez uno de los riesgos que más ha llamado la atención y ha sido objeto de una mayor consideración tanto por la normativa como la doctrina es el vinculado a la protección de los datos personales (Guichot Reina 2009); (Valero Torrijos y Fernández Salmerón 2006). Otro riesgo está vinculado a la eficacia y el propio funcionamiento de las administraciones públicas lo que exige establecer algunos límites a la transparencia o al uso de las TIC para canalizarla. Otro a un exceso de información que puede surgir por la puesta a disposición de los ciudadanos de mucha información. Otro riesgo relacionado con el uso que se pueda hacer de la información y las críticas que se pueden derivar de ello para las administraciones públicas (A. W. Fung, David 2010), así como la falta de capacidad para entender o interpretar la información a la que se tenga acceso. Finalmente, otro riesgo está vinculado al surgimiento de asimetrias informativas.

En efecto, a pesar de la variedad de mecanismos a través de los que se puede canalizar la transparencia administrativa, pueden surgir dificultades para acceder de forma generalizada a la información del sector público o que la información a la que se ha accedido no sea de calidad. Como ha apuntado Schmid-Assmann, "no necesariamente el aumento de información disponible conduce a unas mayores cotas de libertad. Más bien se crean nuevas asimetrías que obligan a plantearse si no existe una responsabilidad del estatal por la procura de información" (Schmidt-Assmann 1998, 292).

Por ello, el derecho puede contribuir decididamente para disminuir las asimetrias informativas garantizando los mínimos que faciliten la máxima transparencia necesaria para todos los ciudadanos, concretando qué información se debe difundir a través de medios electrónicos y delimitando mejor los casos en que la Administración pública no facilitará el acceso a la misma. En este sentido, se puede facilitar una mayor transparencia regulando adecuadamente los diferentes límites a los que se ven sometidos los distintos mecanismos de transparencia y superar de este modo la amplia discrecionalidad con la que en muchos casos se ha regulado esta cuestión.

A la vista de lo anterior se impone la necesidad de impulsar normas jurídicas que regulen de forma conjunta y unitaria la transparencia administrativa, dando respuesta a los diferentes problemas que se han ido observando a lo largo de las páginas anteriores y facilitando la puesta a disposición de los ciudadanos de la información del sector público y reconociendo el papel que los ciudadanos pueden realizar para canalizar la transparencia administrativa. Por todo ello, a nuestro entender, se debería avanzar hacia la convergencia de los regímenes jurídicos vinculados a los mecanismos de transparencia administrativa y superar la 
pluralidad y diversidad de normas que existen actualmente en España, pero también en otros países, regulando los diferentes mecanismos de transparencia administrativa. Estas normas deberían basarse decididamente en las TIC como instrumento a través del que canaliza la transparencia administrativa aunque, lógicamente, no como mecanismo exclusivo.

En particular, la regulación de la transparencia administrativa debería potenciar una mayor difusión de la información a través de medios electrónicos que se pueda ver garantizada en última instancia por un acceso más eficaz a los documentos $y$, finalmente, unas mayores facilidades para reutilizar dicha información como mecanismo para implicar a los ciudadanos en la transparencia administrativa. A tal fin, en primer lugar, se podría especificar qué información, a través de qué canales y con qué calidad se deberá difundir, así como definir cuáles son las obligaciones y responsabilidades respecto a dicha información tanto a nivel interno (por ejemplo, creando un órgano a quien atribuir especificamente esta competencia como sucede en el Reino Unido), como a nivel externo (por ejemplo, fomentando o concretando el alcance de la participación ciudadana en la transparencia administrativa). En segundo lugar, se podrian concretar mejor las excepciones al derecho de acceso como mecanismo de garantía de la transparencia ante aquellos casos en que la Administración pública no haya difundido previamente una información y articular mecanismos ágiles tanto para canalizar el acceso a la información como para hacer frente a eventuales negativas. En tercer lugar, se debería fomentar la reutilización circunscribiendo claramente los casos en los que sería necesario obtener una autorización previa y concretando las condiciones en las que se deberá desarrollar.

Además, las administraciones públicas deberian impulsar políticas tanto orientadas a reducir la brecha digital o establecer infraestructuras tecnológicas adecuadas que garanticen el adecuado acceso a la información (Margetts, $\mathrm{H}$. 2006, 206); (Zeno-Zencovich 2006, 8), como, en general, el incremento de la transparencia administrativa.

En particular, seria oportuno definir políticas que impulsasen el cambio de la cultura ínsita en la mayoría de las administraciones públicas a través del impulso de diferentes fuerzas motoras. Tal y como ha sido reconocido, uno de los principales límites de la mejora de la transparencia administrativa junto a su regulación consiste en la falta de una cultura administrativa orientada a la misma que supere al tradicional secreto que ha caracterizado durante años al modelo burocrático de Administración pública (Sommerman 2010, 18); (OECD 2010, 13).

Para poder avanzar hacia una nueva cultura administrativa, siguiendo a Tim Berners-Lee, se pueden identificar tres grupos de actores que deben participar activamente en esta evolución: ciudadanos, empleados públicos y los directivos públicos (Hogge 2010, 7). Todos ellos deben contribuir decididamente a este proceso ya que, como apunta el inventor de Internet, a la vista de la experiencia norteamericana y británica "se debe empezar por arriba, se debe empezar por en medio y se debe empezar por abajo" (Hogge 2010).

En primer lugar, como ya se ha visto, las TIC apuntan hacia el surgimiento de un nuevo paradigma en la transparencia administrativa caracterizado por la intervención de los ciudadanos (Acar et al. 2009). La propia sociedad se convierte en un actor de la transparencia. Las TIC cambian los flujos de información en la sociedad hacia un nuevo equilibro entre la sociedad civil, las empresas y las administraciones públicas (Parycek y Sachs 2010, 9). De hecho, las TIC están contribuyendo al surgimiento de dos tipos de ciudadanos, los que acceden a la información del sector público y los que, además, la procesan y agregan a otra información para crear nueva información de valor añadido $u$ ofrecer nuevos servicios (Mayo y Steinberg 2007, 11); (Fages y Sangüesa 2008). En este proceso, la ciudadanía debe estar impulsada por un pequeño grupo motivado de hackers cívicos (Hogge 2010).

El impacto de este cambio es significativo $y$, de hecho, recoge la manera de proceder de los ciudadanos cuando buscan información del sector público. No se puede olvidar que actualmente cuando los ciudadanos buscan información del sector público lo hacen en primera instancia en un buscador generalista como Google o Yahoo en vez de acudir a un portal de una administración pública (Margetts, Helen y Escher 2007). Así lo demuestra el Barómetro de marzo de 2009 del CIS, según el cual entre los ciudadanos que utilizan Internet cuando necesitan información del sector público, el 68,9\% utiliza un buscador general (Google, Yahoo, etc.), mientras que el 4,8\% lo hacen a través del portal de información general de la Administración (060) 
y el $24,3 \%$ mediante la página del organismo responsable del servicio.

Esta situación emergente ha llevado a autores como Fung, Graham y Weil a hablar de transparencia colaborativa como "una nueva generación de transparencia que está emergiendo facilitada por el uso de las TIC en la medida en que emprendedores, activistas, reguladores y ciudadanos inventan nuevas maneras de recoger, procesar y distribuir información" (Fung et al. 2007, 152).

Los empleados públicos tienen también un papel muy relevante en la evolución que se está comentando ${ }^{17}$. Al respecto no se puede olvidar que tradicionalmente se había justificado el secreto administrativo en la necesidad de apartar a los empleados públicos de la influencia de los ciudadanos (Castells Arteche 1984, 137). Sin embargo, ahora la situación es bien diferente. El cambio de la cultura organizativa se erige como base para la efectiva transparencia a través de las TIC. De hecho, se propone ir hacia una cultura de la transparencia (Stiglitz 2003) y del gobierno abierto ${ }^{18}$.

Finalmente, los directivos públicos deben liderar el proceso a través de la adopción de un marco regulatorio que facilite que las TIC contribuyan decididamente a la transparencia administrativa. Ya existen algunas iniciativas en esta materia que pueden ilustrar el necesario liderazgo. Se ha hecho referencia a la Directiva de Gobierno Abierto del gobierno norteamericano ${ }^{19}$. Más cerca, la Unión Europea en su Declaración de Mälmo, adoptada en la V Cumbre Ministerial sobre administración electrónica celebrada en noviembre de 2009, apunta la necesidad de incrementar la disponibilidad de información del sector público para su reutilización de acuerdo con lo previsto en la Directiva 2003/98/CE así como de fortalecer la transparencia de los procesos administrativos como base para promover la rendición de cuentas y la confianza en los poderes públicos ${ }^{20}$.

Por todo lo anterior, podemos concluir que las TIC tienen un impacto significativo en el incremento de la transparencia administrativa. Al menos, por lo que se refiere al incremento de sujetos que la canalizan, al objeto de la transparencia, el incremento de mecanismos a través de los que ésta se canaliza y la ampliación de sus finalidades.

Sin embargo, siguiendo a Florini, no debe caerse en el determinismo tecnológico y afirmar que nos encontramos ante una sociedad transparente porque las tecnologías están dificultando esconder la información, puesto que existen otros elementos que inciden en este proceso (Florini $2007,5)$. Tal y como se ha apuntado anteriormente, es necesario evolucionar hacia una cultura de la transparencia. En este proceso y sobre la base del desarrollo tecnológico existente puede tener un papel significativo la adopción de un marco jurídico que determine, concrete e impulse el impacto de las TIC en la transparencia administrativa y en los diferentes mecanismos a través de los que se canaliza, para que gracias a la misma los ciudadanos no sólo puedan ver el interior de las oficinas públicas sino que puedan entrar en ellas y participar activamente en el proceso de difusión de la información del sector público.

De este modo, las TIC podrán contribuir significativamente para conseguir que la transparencia administrativa tenga un impacto político, social y económico.
Recibido: 7 de noviembre de 2011

Aceptado: 25 de febrero de 2012

\section{NOTAS}

* Durante el proceso de revisión de las galeradas se ha tenido conocimiento del anteproyecto de ley de transparencia, acceso a la información y buen gobierno presentado por el gobierno español que, en términos generales, incorpora buena parte de los aspectos apuntados a lo largo del texto. Accesible en: www.leydetransparencia.gob. es (última consulta: julio 2012).
1 Además, en los últimos años el principio de transparencia ha sido incluido en diferentes declaraciones institucionales vinculadas a la reforma y modernización de las administraciones públicas (como, por ejemplo, el Plan Moderniza de la AGE).

2 A lo largo del trabajo únicamente se hará referencia a los mecanismos generales y no a los vinculados a las decisiones en particular 
dirigidos a facilitar información a los interesados en las decisiones administrativas que les afectan, como la motivación de los actos administrativos o la participación de los interesados en el procedimiento administrativo.

3 Véase al respecto el Informe sobre las solicitudes y casos en seguimiento elaborado en 2008 por Access Info Europe en relación con la legislación española actual y la práctica de la Administración en España (http:// www.access-info.org/documents/ files/CUANDO_LO_PUBLICO_NO_ES_ PUBLICO_Def.doc), así como el que unos años antes había impulsado la Open Society Justice Initiative junto con Sustentia sobre el acceso a la información en España (http://www. sustentia.com/transparencia_y_silencio_espana.pdf) (última consulta: noviembre 2011).

4 Accesible en: http://www.whitehouse. gov/the_press_office/Freedom_of_ Information_Act/ (última consulta: noviembre 2011).

5 La Coalición pro acceso ha sido una de las entidades más activas reclamando una nueva ley de acceso a la información pública que cumpla con los nueve principios que se derivan del estudio comparado de la legislación de más de 80 países y de la Convención del Consejo de Europa http://www.proacceso.org/ (última consulta: noviembre 2011).

6 Véase en general el capítulo II del anteproyecto de ley de transparencia, acceso a la información y buen gobierno.

7 Debe tenerse en cuenta la normativa en materia de contratos del sector público (en particular, Ley 30/2007, de 30 de octubre, de Contratos del Sector Público), urbanismo o subvenciones que de manera más o menos explícita incluyen la obligación de difundir información por parte de las administraciones públicas competentes.

Asimismo, el anteproyecto de ley de transparencia y acceso de los ciudadanos a la información pública difundido en julio de 2011 (citado en nota anterior) preveía en su artículo 3 que "Los sujetos incluidos en el ámbito de aplicación de esta Ley facilitarán, preferentemente por medios electrónicos, a través de sus respectivas sedes electrónicas y páginas web, la información cuya divulgación resulte de mayor relevancia para garantizar la transparencia de su actividad".

7 bis Resulta asimismo de interés la lectura de la Ley Foral 11/2012, de 21 de junio, de la Transparencia y del Gobierno Abierto.

8 Véase al respecto, aunque correspondientes a 2008, las estadísticas de uso del portal Gencat de la Generalitat de Catalunya: Accesible en: http://www.gencat.cat/nougencat/ cas/estadistiques.htm (última consulta: noviembre 2011).

9 De hecho, la propia LRISP establece que "la aplicación de esta ley se hará sin perjuicio del régimen aplicable al derecho de acceso a los documentos y a las especialidades previstas en su normativa reguladora".

10 Se toma la definición propuesta por el Public Sector Transparency Board en junio de 2010 (http://data.gov.uk/ blog/new-public-sector-transparency-board-and-public-data-transparency-principles) (última consulta: noviembre 2011).

11 http://www.opengovdata.org/ home/8principles (última consulta: noviembre 2011).

11 bis De acuerdo con la Ley Foral 11/2012, de 21 de junio, de la Transparencia y del Gobierno Abierto, el gobierno abierto es la "forma de funcionamiento de la Administración Pública capaz de entablar una permanente conversación con los ciudadanos y ciudadanas con el fin de escuchar lo que dicen y solicitan, que toma sus decisiones centrándose en sus necesidades y preferencias, que facilita la participación y la colaboración de la ciudadanía en la definición de sus políticas y en el ejercicio de sus funciones, que proporciona información y comunica aquello que decide y hace de forma transparente, que se somete a criterios de calidad y de mejora continua, y que está preparado para rendir cuentas y asumir su responsabilidad ante los ciudadanos y ciudadanas a los que ha de servir".

12 Véase al respecto el Memorando sobre Transparencia y Gobierno Abierto y la Directiva sobre Gobierno Abierto así como el informe de seguimiento sobre su implementación elaborados en 2009, accesibles en: http://www. whitehouse.gov/open (última consulta: noviembre 2011).

13 Como muestra de la extensión de proyectos de apertura de datos públicos en el mundo puede consultarse: http://datos.fundacionctic. org/sandbox/catalog/faceted/; http://epsiplatform.eu/content/category-1-public-sector-information-psi-data-catalogues-governments-direct-access-data (última consulta: noviembre 2011).

14 Acuerdo del Consejo de Gobierno Vasco de 29 de diciembre de 2009. Véase en http://opendata.euskadi. net/ (última consulta: noviembre 2011).

15 Resolución de 2 de febrero de 2009, de la Consejería de Administraciones Públicas y Portavoz del Gobierno, por la que se crea el Repositorio Insti- 
tucional del Principado de Asturias. Véase en: risp.asturias.es/ (última consulta: noviembre 2011).

16 Véase como ejemplo http://data.gov. uk/code-conduct (última consulta: noviembre 2011).

17 A pesar de que en el texto se utiliza la expresión "empleados públicos" se debe concretar que Berners-Lee se refiere a aquellos empleados públicos calificados situados en niveles intermedios de la administración pública implicados y dotados de recursos ("engaged and well-resourced middle layer of skilled government bureaucrats") (Hodge, 2010).

18 Véase la Directiva sobre el Gobierno Abierto.

19 En Australia la Declaración sobre el Gobierno Abierto, que se basa en los principios de informar, implicar y participar, prevé en relación a la información "el fortalecimiento de los derechos ciudadanos de acceso a la información, estableciendo una cultura a favor de la divulgación en todas las agencias del gobierno australiano en particular mediante la innovación en línea, y hacer que la información gubernamental sea más accesible y utilizable", accesible en: http://agimo. govspace.gov.au/2010/07/16/declaration-of-open-government/ (última consulta: noviembre 2011).

20 Accesible en: http://www.egov2009. se/wp-content/uploads/MinisterialDeclaration-on-eGovernment.pdf (última consulta: noviembre 2011).

En el mismo sentido, Declaración de La Granja adoptada en el V Foro Ministerial Unión Europea-América Latina y el Caribe sobre la sociedad de la información Contenidos digitales para una sociedad digital (marzo de 2010), accesible en: http://www. mityc.es/telecomunicaciones/Presidencia/actos/marzo2010/Documents/
ConclusionesForoES.pdf (última consulta: noviembre 2011). Previamente, la Recomendación de la OCDE para un mejor acceso y más efectivo uso de la información del sector público y las administraciones públicas. Accesible en: http://www.oecd.org/ dataoecd/0/27/40826024.pdf (última consulta: noviembre 2011).

\section{REFERENCIAS BIBLIOGRÁFICAS}

Acar, S., Alonso, J. M. y Novak, K. (2009): Improving access to government through better use of the web: W3C Interest Group Note 12 May 2009.

Access Info Europe y Open Knowledge Foundation (2010): Beyond access: Open governmetn data and the right to reuse.

Ackerman, J. M. y Sandoval-Ballesteros, I. E. (2006): "The global explosion of Freedom of Information Acts", Administrative Law Review, 58 (winter).

Alonso Espinosa, C. (2008): "La información en la Red y el principio de neutralidad tecnológica: la libertad de expresión y la difusión de información administrativa", Revista Vasca de Administración Pública, 81 (mayo-agosto).

Apfelroth, J. (2006): "The open government act: a proposed bill to ensure the efficient implementation of the freedom of information act", Administrative Law Review, 58 (winter).

Arena, G. (1996): "Transparenza amministrativa e democrazia". En Berti, G. y De Martín, G. C. (eds.), Gli istituti della democrazia amministrativa. Atti del seminario di studio-Roma, 12 febbraio 1993, Milán, Giuffrè Editore.

Arena, G. (2008): "Le diverse finalità della trasparenza amministrativa". En MerIoni, F. (ed.), La trasparenza amministrativa, Milano, Giuffrè.
Bae Brandtzæg, P. y Lüders, M. (2010): eCitizen2.0: The ordinary citizen as a supplier of public-sector information, Oslo: Ministry of Government Administration and Reform.

Berners-Lee, T. (2009): Putting Government Data online.

Birkinshaw, P. (2006): "Freedom of Information and Openness: Fundamental Human Rights?", Administrative Law Review, 58 (1).

Blasco Díaz, J. L. (2010): "El sentido de la transparencia administrativa y su concreción legislativa". En R. García Macho (ed.), Derecho administrativo de la información y administración transparente, Madrid, Marcial Pons.

Calderón, C. y Lorenzo, S. (2010): "Introducción". En Calderón, C. L., Sebastián (ed.), Open Government: Gobierno Abierto, Alcalá la Real, Algón.

Carloni, E. (2005): "Nuove prospettive della trasparenza amministrativa: dall'acceso ai documenti alla disponibilità delle informazioni", Diritto Pubblico, 2.

Carloni, E. (2008): "Gli strumenti della trasparenza nel sistema amministrativo italiano e la sua effettività: forme di conoscibilità, quantità e qualità delle informazioni". En Merloni, F. (ed.), La trasparenza amministrativa, Milano, Giuffrè.

Castells Arteche, J. M. (1984): "El derecho de acceso a la documentación de la administracion pública", Revista de Administración Pública, 103.

Cavaleri, P. y Venturini, F. (2004): Documenti e dati pubblici sul web. Guida all'informazione di fonte pubblica in rete, Bologna, II Mulino.

Cerrillo i Martínez, A. (2005): "E-Información: hacia una nueva regulación del acceso a la información", Revista Internet, Derecho y Política, 1.

Cerrillo i Martínez, A. (2009a): "La difusión de información de la administración 
local a través de medios electrónicos". En Cerrillo i Martínez, A. y Galán Galán, A. (eds.), Informe sobre la administración electrónica local, Barcelona, Fundació Carles Pi i Sunyer.

Cerrillo i Martínez, A. (2009b): "La reutilització de la informació del sector públic a Espanya", Lligall, 29.

Cerrillo i Martínez, A. (2010a): "La administración electrónica en el derecho autonómico comparado", Revista Vasca de Administración Pública, 86 (enero-abril).

Cerrillo i Martínez, A. (2010b): "La difusión de información pública a través de medios electrónicos: claroscuros de la ley de acceso electrónico de los ciudadanos a los servicios públicos". En Cotino, L. (ed.), La ley de administración electrónica, València, Tirant lo Blanch.

Cerrillo i Martínez, A. (2011): "The regulation of diffusion of public sector information via electronic means: Lessons from the Spanish regulation", Government Information Quarterly, 28.

Cerrillo i Martínez, A. (2012): "How can law improve the use of web 2.0 in public sector information diffusion?" International Journal of Electronic Governance.

Cerrillo i Martínez, A. (2000): L'aplicació del dret d'accés a la documentació administrativa de la Generalitat de Catalunya, Barcelona, Escola d'Administració Pública de Catalunya-Generalitat de Catalunya.

Cerrillo i Martínez, A. y Galán Galán, A. (2007): Qualitat i responsabilitat en la difusió d'informació pública a Internet, Barcelona, Escola d'Administració Pública de Catalunya i Generalitat de Catalunya.

Chang, A.-M. y Kannan, P. K. (2008): Leveraging Web 2.0 in Government, Washington, IBM Center for The Business of Government.
Chevalier, J. (2007): Science administrative

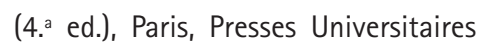
de France.

Cluzel-Métayer, L. (2006): Le service public et l'exigence de qualité, Paris, Dallow.

Coglianese, C. (2009): "The Transparency President? The Obama Administration and Open Government", Governance: An International Journal of Policy, Administration, and Institutions, 22 (4).

Comisión Europea (1998): "La información del sector público: un recurso clave parar Europa". Libro Verde sobre la información del sector público en la sociedad de la información. COM (1998) 585.

Comisión Europea (2001): La gobernanza europea: un libro blanco. COM(2001) 428 final

Comisión Europea (2009): Comunicación de la Comisión al Parlamento Europeo, al Consejo, al Comité Económico y Social Europeo y al Comité de las Regiones - La Reutilización de la información del sector público - Revisión de la Directiva 2003/98/CE. COM(2009) 212 final.

Cotino Hueso, L. (2007): "Retos jurídicos y carencias normativas de la democracia y la participación electrónicas". Revista Catalana de Dret Públic, 35.

Curtin, D. y Meijer, A. J. (2006): "Does transparency strenghen legitimacy?", Information Polity, 2.

Darbishire, H. (2010): Proactive transparency: The future of the rigth to information?, Washington: The World Bank.

Dawes, S. S. (2010): "Stewardship and usefulness: Policy principles for information-based transparency". Government Information Quarterly, 27.

Dekkers, M.; Polman, F.; Velde, R. T. y Vries, M. D. (2006): MEPSIR. Measuring European Public Sector Information Resources.

Dror, Y. (2000): "Transparency and openness of quality democracy". En Kelly, M. (ed.), Openness and Transparency in Governance: Challenges and Opportunities: NISPAcee and EIPA.

Fages, R. y Sangüesa, R. (2008): "Good practice exchange from a Web 2.0 point of view", European Journal of ePractice, 1.

Fernández Ramos, S. (1997): El derecho de acceso a los documentos administrativos, Madrid, Marcial Pons.

Fenster, M. (2010): "Seeing the State: Transparency as Metaphor", Administrative Law Review, 62 (3).

Florini, A. (2007): "The batlle over transparency". En Florini, A. (ed.), The right to know. Transparency for an open world, New York, Columbia University Press.

Fornefeld, M., Boele-Keimer, G., Recher, S. y Fanning, M. (2009): Assessment of the Re-use of Public Sector Information (PSI) in the Geographical Information, Meteorological Information and Legal Information Sectors, Düsseldorf, MICUS.

Frost, A. (2003): "Restoring faith in government: transparency reform in the United States and the European Union", European Public Law, 9 (1).

Fung, A. (2009): "Defining transparency", Nextgov.

Fung, A.; Graham, M. y Weil, D. (2007): Full disclosure. The perils and promise of transparency, New York, Cambridge University Press.

García Macho, R. (2010): "El derecho a la información, la publicidad y la transparencia en las relaciones entre la administración, el ciudadano y el público". En R. García Macho (ed.), Derecho administrativo de la información y administración transparente, Madrid, Marcial Pons.

Gentot, M. (1994): "La transparence de l'administration publique", Revue des Sciences Administratives.

Grimmelikhuijsen, S. (2009): "Do transparent government agencies strengthen trust?", Information Polity, 14. 
Guadián, C. (2010): "¿Transparencia?" En Calderón, C. y Lorenzo, S. (eds.), Open Government: Gobierno Abierto, Alcalá la Real, Algón.

Guichot Reina, E. (2009): Publicidad y privacidad de la información administrativa, Madrid, Thomson-Civitas.

Hazell, R. y Worthy, B. (2010): "Assessing the performance of freedom of information", Government Information Quarterly, 27.

Heald, D. (2006a): "Transparency as an instrumental value". En Hood, C. y Heald, D. (eds.), Transparency. The key to better governance? Oxford, Oxford University Press for The British Academy.

Heald, D. (2006b): "Varieties of Transparency". En Hood, C. y Heald, D. (eds.), Transparency. The key to better governance?, Oxford, Oxford University Press for The British Academy.

Herz, M. (2009): "Law lags behind: FOIA and affirmative disclosure of information", Cardozo Public Law, Policy and Ethics Journal, Summer.

Hogge, B. (2010): Open Data Study. Commissioned by the Transparency and Accountability Initiative.

Kubicek, H. (2004): "Third-generation Freedom of Information in the context of e-Government: the case of Bremen, Germany". En Aichholzer, G. y Burkert, H. (eds.), Public Sector Information in the Digital Age. Between markets, public management and citizens' rights, Cheltenham, UK, Edward Elgar.

Lessig, L. (2010): "Against transparency: The perils of openness in Government". En Gøtze, J. y Bering Pedersen, C. (ed.), State of eUnion: Government 2.0 and Onwards, 21Gov.net.

Llinares, J. (2010): "Las 10 claves para entender la colaboración en el Modelo Open Government". En Calderón, C. L., Sebastián (ed.), Open Government: Gobierno Abierto, Alcalá la Real, Algón.
Malaret i Garcia, E. (2007): "Els serveis públics informacionals: l'emergència de nous serveis públics en la societat de la informació i del coneixement", Revista Catalana de Dret Públic, 35.

Margetts, H. (2006): "Transparency and digital government". En Hood, C. y Heald, D. (eds.), Transparency. The key to better governance? Oxford, 0xford University Press for The British Academy.

Margetts, H. y Escher, T. (2007): Understanding Governments and Citizens On-line: Learning from E-commerce, Paper presented at the Paper presented at the annual meeting of the American Political Science Association.

Martínez Gutiérrez, R. (2009): Administración pública electrónica, Madrid, Civitas-Thomson Reuters.

Mattarella, B. G. (2005): "Profili Generali". En Mangaro, F. y Romano Tassone, A. (eds.), I nuovi diritti di cittadinanza: il diritto d'informazione, Torino, G. Giappichelli Editore.

Mayo, E. y Steinberg, T. (2007): The Power of Information: An independent review, Londres, Office of Public Sector Information.

Meijer, A. (2009): "Understanding modern transparency", International Review of Administrative Sciences, 75 (2).

Mendel, T. (2008): Freedom of Information: A Comparative Legal Survey (2. ${ }^{\text {a ed.), }}$ Paris, UNESCO.

Merloni, F. (2005): Introduzione all'eGovernment. Pubbliche amministrazioni e società dell'informazione, Torino, G. Giappichelli Editore.

Merloni, F. (2008): "Trasparenza delle istituzioni e principio democratico". En Merloni, F. (ed.), La trasparenza amministrativa, Milano, Giuffrè.

Mestre Delgado, J. F. (1998): El derecho de acceso a archivos y registros administrativos: [Análisis del artículo 105.b) de la Constitución] (2. a ed.), Madrid, Civitas.
Michener, G. (2009): The surrender of secrecy? Explaining the strength off transparency and access to information laws, Paper presented at the 2009 annual meeting. Retrieved from http://papers.ssrn.com/sol3/Delivery. cfm/SSRN_ID1459098_code1320170. pdf?abstractid $=1449170$ \& mirid $=1$

Mock, W. (1999): "On the centrality of information law: a rational choice discussion of information policy and transparency", Journal of Computer \& Information Law, 17.

Molnar, P. (2009): "The paradox of informed participation: What universities can do for freedom of information", Cardozo Public Law, Policy and Ethics Journal, Summer.

Moser, C. (2001): How open is 'open as possible'? Three different approaches to transparency and openness in regulating access to EU documents, Vienna, Institute for Advanced Studies.

OCDE (2001): Citizens as partners. Information, consultation and public participation in policy-making, Paris, OCDE.

OCDE (2006): Digital broadband content: Public Sector Information and Content, Paris, OCDE.

OCDE (2007): Participative web: User-created content, Paris, OCDE.

OCDE (2010): "The Right to Open Public Administrations in Euroep: Emerging Legal Standards", Sigma Papers (Vol. 46), Paris, OCDE.

Oliver, R. W. (2004): What is transparency? New York, McGraw-Hill.

Openthegovernment.org (2010): Secrecy Report Card 2009: Indicators of secrecy in the Federal Government, Washington, Openthegovernment. org.

Orofino, A. G. (2005): "La pubblicità telematica nell'impianto del Codice dell'amministrazione digitale", Informatica e diritto, 1-2. 
Parada Vázquez, R. (1993): La Ley de Régimen Jurídico de las Administraciones Públicas, Madrid, Marcial Pons.

Parycek, P. y Sachs, M. (2010): "Open government-information flow in Web 2.0", European Journal of ePractice, 9 (march).

Pas, J. (2002): "The commercialization of government information and the proposar for a Directive (2002) 207 by the European Commission", E-law- Murdoch University Electronic Journal of Law, 9 (4).

Pigrau Solé, A. (2008): Acceso a la información, participación pública y acceso a la justicia en materia de medio ambiente: diez años del Convenio de Aarhus, Barcelona, Atelier.

Piotrowski, S. J. y Van Ryzin, G. G. (2007): "Citizen attitudes toward transparency in local government", The American Review of Public Administration, 37 (3).

Pira International (2000): Commercial exploitation of Europe's public sector information, Final Report. ${ }^{\circ}$

Prat, A. (2006): "The more closely we are watched". En Hood, C. y Heald, D. (eds.), Transparency. The key to better governance? Oxford, Oxford University Press for The British Academy.

Privacy International (2006): Freedom of Information around the World 2006. A Global Survey of Access to Government Information Laws.

Puybasset, M. (2003): "Le droità l'information administrative", Actualité Juridique Droit Administrative, 25.

Ramos Simón, F. (2003): "La reutilización de la información del sector público. Aproximación del contenido de la propuesta de directiva 2002", Revista General de Información y Comunicación, 13 (2).

Rams Ramos, L. (2008): El derecho de acceso a archivos y registros administrativos, Madrid, Reus.

Razquin Lizarraga, J. A. y Ruiz de Apodaca Espinosa, Á. (2007): Información, participación y justicia en materia de medio ambiente. Comentario sistemático a la Ley 27/2006, de 18 de julio, Cizur Menor, Thomson-Aranzadi.

Rivero, J. (1989): "La transparence administrative en Europe-Rapport de synthèse", Annuaire Européen d'Administration Publique.

Roberts, A. (2006): "Governmental adaptation to transparency rules". En Hood, C. y Heald, D. (eds.), Transparency. The key to better governance? Oxford, Oxford University Press for The British Academy.

Robinson, D.; Yu, H.; Zeller, W. P. y Felten, E. W. (2009): "Government data and the invisible hand", Yale Journal of Law \& Technology, fall.

Sainz Moreno, F. (2004): "Secreto y transparencia". En AAW, Estudios para la reforma de la administración pública, Madrid, INAP.

Sánchez Morón, M. (1991): El control de las administraciones públicas y sus problemas, Madrid, Tecnos.

Schmidt-Assmann, E. (1998): La teoría general del Derecho administrativo como sistema (edición 2003), Madrid, INAPMarcial Pons.

Sommerman, K. P. (2010): "La exigencia de una administración transparente en la perspectiva de los principios de democracia y del estado de derecho". En R. Garcia Macho (ed.), Derecho administrativo de la información y administración transparente, Madrid, Marcial Pons.

Stiglitz, J. E. (2003): "On liberty, the right to know, and public discourse: the role of transparency in public life". En Gibney, M. J. (ed.), Globalizing rights, Oxford, Oxford University Press.

Stiglitz, J. E. (2007): "Foreword". En Florini, A. (ed.), The right to know. Transparency for an open world, New York, Columbia University Press.

Sucevic, P. (2008): "De l'accès à la reutilisation: le nouveau régime applicable aux donnees publiques en France". En Ponti, B. (ed.), Il regime dei dati pubblici. Esperienze europee e ordinamento nazionale, Santarcangelo di Romagna, Maggioli.

Valero Torrijos, J. (2009): "Acceso a los servicios y a la información por medios electrónicos". En Gamero Casado, E. y Valero Torrijos, J. (eds.), La Ley de Administración electrónica. Comentario sistemático a la Ley 11/2007, de 22 de junio, de acceso electrónico de los ciudadanos a los Servicios Públicos (2. ed.), Cizur Menor, Thomson-Aranzadi.

Valero Torrijos, J. y Fernández Salmerón, M. (2006): "Protección de datos personales y Administración electrónica", Revista Española de Protección de Datos, 1.

Vladeck, D. C. (2008): "Information accesssurveying the current legal landscape of federal right-to-know laws", Texas Law Review, June.

Zeno-Zencovich, V. (2006): "II 'diritto ad essere informati' quale elemento del rapporto di cittadinanza". Rivista di Diritto dell'Informatzione e dell'Informatica, 1. 